\title{
Nacer en la Liquidez
}

\section{Born in Liquidity}

TIPO DE TRABAJO: Comunicación.

\section{PALABRAS CLAVE}

Tiempos líquidos, cuerpo femenino, relaciones, diosas primigenias, miedo.

\section{KEY WORDS}

Liquid times, female body, relationships, primitive godesses, fear.

\section{RESUMEN}

Debido al rampante desarrollo tecnológico, y los cambios en la forma de entender nuestro día a día, han surgido nuevos abordajes explicando el ahora, la "liquidez" y la "ligereza", términos acuñados por Gilles Lipovetzky y Zygmunt Bauman, son una constante en diversas áreas del conocimiento, filosófico, artístico, sociológico, tecnológico incluso económico. El lenguaje se transforma por una necesidad, que refleja un profundo cambio en la sociedad.

En este trabajo se analiza como estas descripciones de nuestra realidad, como "fake news", "binge wathching", "retropia", "bullying", "polite sex", "pendulum liberty", y, específicamente como características de las Diosas primigenias y el imaginario femenino de nuestros días, se retoman con fuerza en ámbitos de las artes plásticas, la mass media y la economía rampante.

Hemos clasificado a las generaciones como baby boomers (1946 - 1964), millennials generación Y, generación X (1980-2000) y ahora la generación líquida (2000-2019) y diferenciamos los rápidos cambios en los líquidos.

Como se ve el mundo líquido, por un no líquido y un líquido. Enormes diferencias en la forma de entender el mundo que nos rodea y sus características, en todos los ámbitos, y específicamente, motivo de esta investigación, específicamente se refiere al cuerpo femenino, el amor líquido y sus vínculos con el arte.

Como entender al que ha nacido líquido, su relación con el amor, las relaciones efímeras y como esto se ve reflejado en el arte. Las transformaciones en el entendimiento del cuerpo femenino, como ejemplos señalo el extendido uso del los tatuajes, la cirugía plástica y las costumbres hípsters, como se ve la moda en las nuevas diosas primigenias, las actitudes de la generación líquida ante los vínculos humanos, el análisis de las relaciones on-line, la transformación de la agresividad "el bullying "la inestabilidad emocional y su reflejo en el arte líquido.

\section{ABSTRACT}

Due to the ravaging technological development our world and the way we understand it is changing every day, new approaches to understand this liquidity, liquid times or social lightness have been proposed by philosophers like Gilles Lipovetzky or Zygmunt Bauman, constant changes involve, several areas of knowledge and behavior, in art, philosophy, economy and technology and specially how we see ourselves, How the born liquid generation relates to their environment or surroundings and to their bodies and show social skills are constantly changing. Language shows the way, its transformation reflects a profound change in our society.

Analyzing descriptions like fake news, binge watching, retropia, bullying, polite sex, pendulum liberty and specifically the image of the female body in our times, and how these changes influence even control the esthetical needs of the liquid generation through the invasive mass media and the raging economy.

With the classification of the generations as baby boomers, (1946-1984), millennial generation, $Y$ and $X$ generation (1980-2000) and now the liquid generation (2000-2019) we distinguish the rapid changes now a days. 
How the liquid world works, how it's seen by a nonliquid. Huge differences in the way of reacting, confiding, understanding the world that surrounds us specially the image changes in the female body, it's understanding of private life and the web relationships, liquid love and it's bond to art.

How to understand the liquid born, their relationships, with love, polite sex, short-lived relation and its reflection on art and their bodies. The transformations in the female and male bodies through tattoo, plastic surgery, hipster ways, how fashion is inspired by the primitive goddess bodies and mass media has made them come back, revive in the liquid generation. The on-line relations, the transformation of aggression through bullying and the emotional instability that liquidity cause.

\section{INTRODUCCIÓN}

La comunicación es la base del encuentro, del discernimiento y de la comprensión de conceptos, la forma como entendemos nuestra realidad, la comunicación, mass media en la sociedad y en el arte, en las sociedades líquidas, influye mas que nunca en la generación líquida y se entromete en las vidas líquidas con mas influencia y fuerza que nunca, la convivencia se guía por estas nuevas reglas.

Los tiempos y los significados de las palabras cambian, se transforman y surgen cada día nuevos; ahora mas que nunca cobijados por los avances tecnológicos en que se ven engullida la generación liquida, "los nacidos después del año 2000", (Bauman 2019) como señala Zygmund Bauman y Thomas Leoncini en el ultimó libro publicado por el filosofo polaco.

Esta generación nacida líquida, es con la que tenemos como docentes que tender puentes de comunicación, ideogramas, entender si es que queremos hacer una diferencia y no quedar incomunicados o peor aun, mal comunicados, en un mundo cambiante y liquido, el cual tiene su propio lenguaje y visión de fenómenos sociales, artísticos y de comunicación.

Lo mas parecido a lo que esta sucediendo en este cambio generacional tan brusco es la teoría de la relatividad:

Albert Einstein demuestra en su teoría de la relatividad que el tiempo es relativo, la velocidad es relativa, la localización de los sucesos físico, tanto en el tiempo como en el espacio son relativos al estado de movimiento del observador, es decir la velocidad depende de nuestro movimiento como lo plantea Waldrop, M. (2017)

No encuentro mejor forma de explicar la aceleración social en la que se mueven los nacidos líquidos.

Sociológicamente hablando, la generación líquida se mueve y cambia de pensamiento a la velocidad de la luz (300,000 mil kilómetros por segundo), por continuar con el paralelismo con la teoría de la relatividad, resultado de los avances en la tecnología, de la ciencia y de la economía. Sea cual fuere el motivo nos enfrentamos a un cambio generacional de pensamiento y actitud.

\section{METODOLOGÍA}

Autores como Gilles Lipoveyzky, Zygmunt Bauman, Hartmut Rosa, Byung-Chul Han, Leonidas Donskis entre otros filósofos y sociólogos ya desde hace mas de una década nos han señalado, la evolución de la generación líquida, generación que ha nacido inmersa en la tecnología y sus mutaciones continuas. Acompañado de un sistema económico voraz, "que les hace relacionarse con la realidad en una forma acelerada e inestable"(Lipovetzky 2015 Pag 10).

Este vivir líquido afecta todas las esferas del desarrollo humano; especial interés para este trabajo son los cambios en la visión del cuerpo femenino y las relaciones interpersonales líquidas como se afectado en su relación y estética.

La tecnología ha creado nuevos ídolos, efímeros, poco veraces y líquidos, "trenders", ellos entienden perfectamente el poder de la comunicación y el comercio a través de la tecnología y lo utilizan para ser lideres de opinión, y empoderarse a través del like. No teniendo escrúpulos, "moral blindness " estos trenders pueden ser menores de edad manipulados y alterados en su imagen los que promueven el comercio voraz, entre otros.

Este trabajo cualitativo se adentra en los nuevos significados líquidos y su impacto en los cuerpos de la generación líquida.

Términos como anomia, Burn out síndrome, Peak stuff, Moral blindness, Retropia, Sexting, Like, Black mirror, Liquid fear, Liquid love ,Liquid Economy Bitcoin, Binge Watching, Nanotech, Small bang, Homo eligens, Hats society-Fear society Fake news, Liquid evil, Anomia, Twitteratura, light sex, características la era del HIPER, hiper poder, hiper capitalismo, hiper clase, hiper individualismo, hiper mercado, 
hiper miedo, no solo debemos incluirlos en nuestro lenguaje sino comprenderlos y hacerlos parte de nuestra vida, si es que queremos llevarles el paso a los mutantes líquidos.

\section{DESARROLLO}

La ligereza con la que vive la generación líquida es su principal característica. Cito a Lipovetzky cuando advierte " la ligereza frívola, es una característica primordial del espíritu que nos invade, que nos lleva a la angustia y a la inestabilidad, al miedo Liquido.

Lipovetzky define esta liquidez como formas de vivir esta época. En su libro Ligereza las clasifica en siete diferentes siendo la mas peligrosa la frívola.

Esta descripción de la forma en que la tecnología, las comunicaciones y el comercio afecta a la generación líquida, explica su forma de relacionares y de actuar. Las que nos interesan en este trabajo son la frívola, la de estilo y la veleidosa, sin embargo menciono las demás.

“1.- ligereza aérea, el sueño de elevarse por los cielos es inmemorial, “(Lipovetzky 2015 Pag. 15)

2.- "Ligereza de movilidad, vinculada a la miniaturización de objetos, (small bang, nano tech) conectados a una red, los líquidos permiten vencer las limitaciones temporales y espaciales inventando un nuevo ser nómada, fluido e informático."(Lipovetsky 2015 pag187)

3.- "Ligereza de distracción se refiere a los códigos y practicas sociales destinadas a aligerar el peso de la existencia " 7 huir de la pesantez del mundo, ser trendy, no formar lazos sociales perdurables.

4.- “Ligereza frívola ejemplificada en un inicio por Lipovetzky con la moda.

En lo frívolo de las apariencias, el ahora popular "like", que es capaz entre los jóvenes de incitar cambios de conducta, en el afán de lograr la ansiada popularidad virtual."(Lipovetzky 2015 Pag 187)

El encanto de la imagen, la fachada, el revolotear de idea en idea, sin profundizar en ninguna, no analizar si se trata de una verdad o es un "fake news". Las conversaciones sobre temas intrascendentes o la perdida de tiempo al verse inmersos en un "binge watching" generalizado, ( termino que deriva de las personas adictas a las series televisivas basura, dedicando en ocasiones días enteros a ver estos programas, vacíos de cualquier contenido cultural ).

5.- La ligereza veleidosa aplicada al comercio sexual, las nuevas infidelidades, el casual sex ", la "post-privacy", el sexting, las relaciones por internet, relaciones virtuales.

El sexting es un ejemplo de comportamiento líquido derivado del advenimiento y uso de las tecnologías por los nacidos líquidos. Comportamiento de la comunicación considerado "normal", en donde el liquido abandonado a la "nube", provoca una intervención en lo privado como nunca antes se había experimentado.

6.- "La ligereza de estilo.- Conectada al estereotipo de belleza, la belleza y la juventud considerada un bien adquirible",(Lipovetzky 2015) la herencia a la generación liquida de "trenders" inmersos en el mercado de las cirugías plásticas, los tatuajes, la

búsqueda de la eterna juventud, llevado de la mano de el comercio de la estética corporal, la industria de la alimentación ( el mercado de la alimentación histérica, lo vegano , packaging responsable, la comida global, los Eenmaal, la desconexión personal del que come solo, prebióticos, los florales, vinagres para el detox, tendencias alimenticias, reforzadas y apoyadas en el mass media y los ídolos de la generación líquida.

Como menciona Byung Chul Han "el poder sobre la muerte es un poder de la vida cuya función no es matar, sino la imposición completa de la vida. El viejo poderío de la muerte cede ante la administración de cuerpos y la gestión calculadora de la vida. “( Han, B.C. 2017)

7.- "La ligereza sabia, vida frívola con la intención de huir hacia placeres que continuamente son renovados a el paso que les marca el mercado,"11 los "gadgets", la "novelity", un ejemplo es la vuelta a lo retro, el cambio constante de ideas religiosas, budismo, Hinduismo, sin realmente conocer la filosofía del pensamiento religioso

Su libro póstumo Zygmunt Bauman lo dedica a los nacidos líquidos, en una serie de entrevistas con Thomas Leoncini, en donde la ligerezas de las que nos advierte Lipovetzky se confirman en este análisis, y como se ven reflejadas en los nacidos líquidos gracias a la HIPER exposición a trenders, medios, y tecnología anomia, inicia con las transformaciones de la piel, skin deep transformations, los 
tatuajes, que los incluye en una forma de mutaciones de la generación liquida que tiene que ver con el control, manejo y alteraciones de su cuerpo, el tatuaje como forma frenética de hacerse notar, en lo publico. Un ejemplo de los públicos mas líquidos de esta generación, los futbolistas, mueven masas, sus motivos altamente económicos, (los nuevos gladiadores) todos ellos tatuados, tatuajes compartidos vidas abiertas a lo publico, y ejemplos a seguir.

Bauman nos advierte sobre el tatuaje, "es un modo de manipular la apariencia publica de sus cuerpos, es un deber que todos esperan, aun cuando los diseños son suministrados por la sociedad, se cree que es una actividad "creativa", fashion" la idea de identidad y auto identificación ha hecho erupción y llena el vacío intentando pertenecer a una clasificación o nivel social." (Bauman 2017 Pag 12)

La identidad del líquido es un trabajo del “do it yourself” esta identidad reconcilia al líquido con un sentido de pertenencia, autodefinición y capacidad creativa, la fragilidad irreparable que los sostiene y mantiene en acción."(Bauman 2017 Pag 10)

El tatuaje en las generaciones líquidas ha hecho del cuerpo su sitio favorito para poner estos signos de esperanza, e auto afirmación, como dice Bauman (2017) "el tatuaje es el milagro de milagros, la posibilidad de escoger libremente la marca que te defina".

Entre los líquidos de vuelve un argumento de pertenencia, entre mas visible mas intrusivo, es un trabajo continuo de representación de nuestros cuerpos en relaciona a la energía que le dedicamos.

En su libro Nacidos Líquidos Baumna (2019) señala, en la generación liquida otra característica es el abuso de la cirugía plástica, según estadísticas del año 2000 al año 2018 han aumentado cirugía de" Busto un 89\%, cirugía de región glútea $252 \%$ y reconstrucción de vaginas 3973\% ( en 2007 se llevaron a cabo 207 operaciones y para 2015 se hicieron 8431 cirugías".

En la liquidez el cuerpo que se ha vuelto un blanco para la identificación del líquido, debe de ser legitimado por los likes por la fashion, la cultura dominante sienta los cánones si no cedes a esta la presión mediática y del like del liquido se ve agredido, "la fashion vuelve legitima a la sinergia de autodestrucción la "humanización de la bello" hasta crear un estereotipo, "lo diferente causa incomodidad". (Han, B.C. 2016)

Tenemos pues el tándem de los líquidos del like y del black mirror, la twiteratura del tatuaje, la cirugía plástica mas la barba, son las llaves de la representación de la historia del fashion y de la creación de una imagen para ser visible públicamente.

Indudablemente la evolución técnica ha influenciado en la forma en que nos relacionamos y como llega a nosotros la información.

En este gráfico publicado por el Diario El País, claramente se ve como cada avance tecnológico ha afectado a los lideres de opinión su visibilidad ha cambiado de la mano de las tecnologías, afectando a la población de todos los tiempos, y refuerza lo que Bauman sostiene los futbolistas y el mercado que se mueve a su costa es el trender de nuestros días. 
Antes de la imprenta

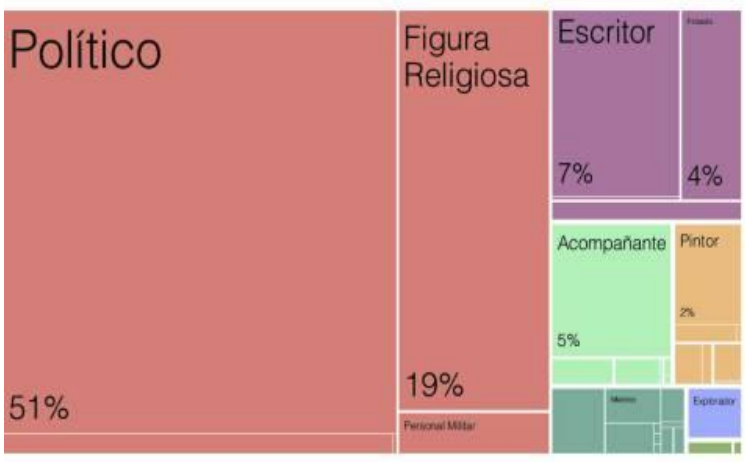

Después de la radio y el cine

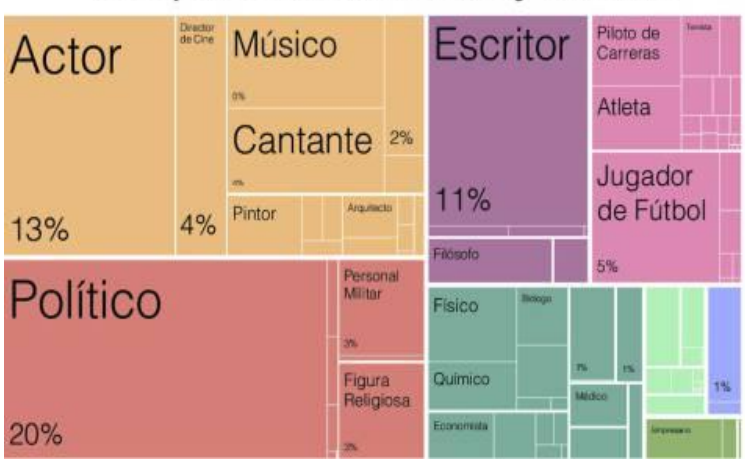

Después de la imprenta

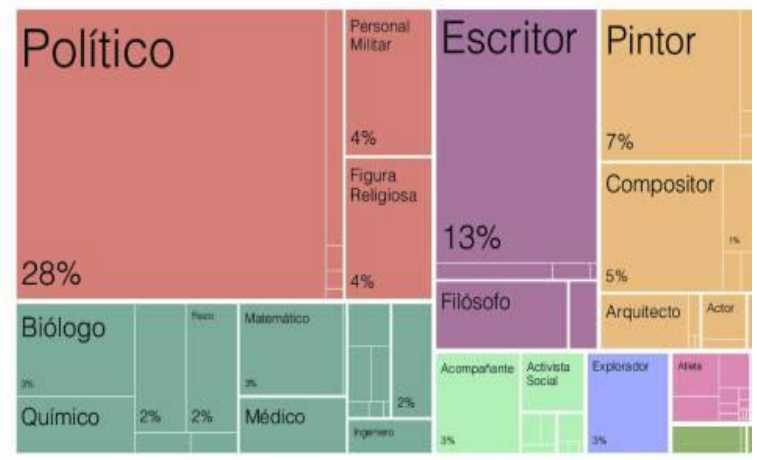

Después de la televisión

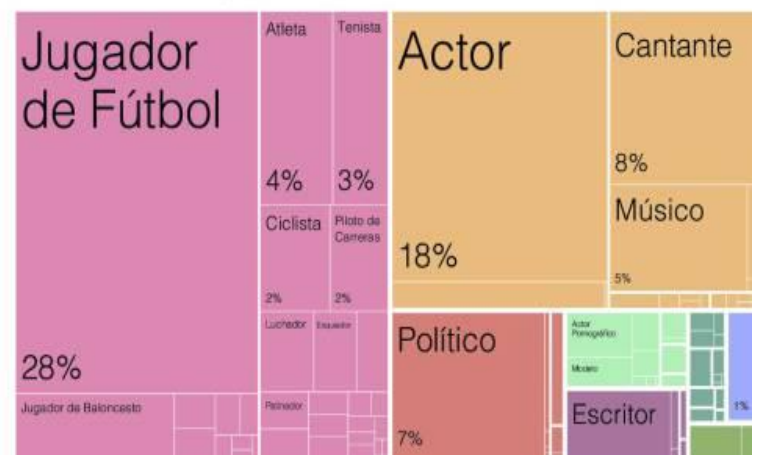

Distribución de las ocupaciones en la base de datos Pantheon 2.0 en cuatro periodos históricos.

La imprenta, la radio o la televisión generaron sus propios famosos

http://elpais.com/el país/2019/02/20/ciencia/1550651024_330137.html JARA FIGUEROA ET AL.

Continuando con este análisis, no solo el tatuaje cambia el cuerpo, la cirugía plástica ha en las últimas décadas transformado los cuerpos de la generación líquida con la presión de los medios de comunicación.

"No hay mejor negocio que el negocio de la cirugía plástica, el sistema de consumo lo exige, "si puedes hazlo, mejora tu apariencia, no hacerlo seria humillante y un golpe doloroso a la auto estima" (Bauman 2017 Pag 20)

Estas máximas hacen que la economía consumista sobreviva, la magia de la estrategia de que la posibilidad de una mejoría es obligatoria y sucede en muchas áreas, la alimentación, la farmacéutica, el turismo incluso en la religión.

Todo pasa a ser, el patrón del outer look y el like, la sociedad Selfie solo la hacen que se vea a si misma buscando la conversión milagrosa que los lleve al éxito.

“Posición social, imagen pública, proclamada a gritos, yo lo puedo pagar" (Bauman 2017 Pag24)

La comunicaciones masivas han alterado también la vida emocional del nacido líquido, "el tabú de encontrar el amor en línea", en el no sitio como lo llama (Bauman 2017 Pag 56).

Esta esfera del individuo se ha visto totalmente revolucionada, el liquido vive en el no lugar, ya no se frecuentan los hoteles, centros comerciales, o se hacen presentaciones tribales, ahora las relaciones de hacen en el NO ESPACIO, en la WEB, en LA LINEA, universos digitales que técnicamente facilitan la comunicación, pero que sin embargo están lejos muy lejos de ser inocuos o seguros.

"La liquidez ha alterado totalmente nuestra infraestructura psicológica y nuestros prototipos de inteligencia kinestésica. Han llevado lo PRIVADO A LO PUBLICO, es un limbo de bolsillo en donde se construyen universos paralelos, un no lugar líquido". 
Esta forma de comunicación ha alterado los esquemas psicológicos del nacido líquido, se esperan resultados inmediatos, La rapidez, la cantidad y la accesibilidad imperan, pero el peligro esta en este "no espacio". Se cree democrático y sin embargo es de un totalitarismo absoluto:" Vemos a la web como el hábitat ideal, también como democrático y político, sin embargo mas que democrático, se parece mucho mas a un totalitarismo, en el que nos sentimos libres para bloquear y eliminar o suprimir a cualquier usuario". (Bauman 2017)

Facebook lo hace posible al bloquear usuarios sin embargo los peligros de los perfiles falsos o la venta de datos no la tiene controlada como nos hacen creer son patentes las dificultades que últimamente ha tenido este tipo de empresa, e inimaginablemente como sin ninguna consecuencia los líquidos siguen confiando en ellas.

Este hábitat del líquido da la falsa sensación de seguridad el estar online y sentirse mas en control que al estar offline, la angustia de no estar conectados, de no tener accesibilidad se asocia a no tener éxito, a no tener likes, a no estar relacionado. Como se ven en las imágenes el fashion nada y se hace cualquier intervención por ser notada, Diosas liquidas?

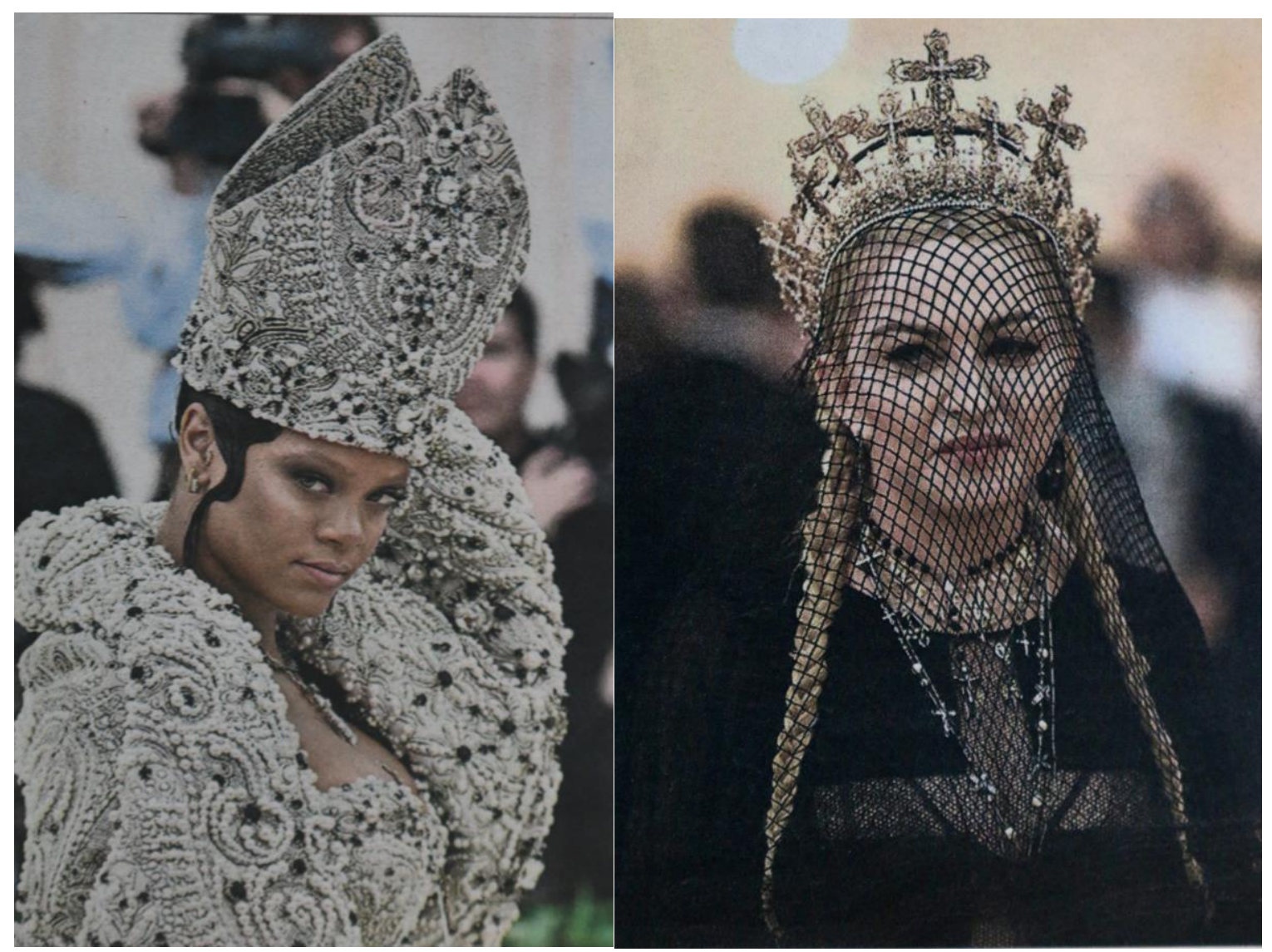

http://elpais.com/el país/2019/02/20/ciencia/1550651024 330137.html JARA FIGUEROA ET AL.

"Internet en lugar de dar el servicio de extender el volumen y elevar la calidad de la integración humana, el entendimiento mutuo, la cooperación y la solidaridad, internet ha facilitado practicas de cerrazón, separación, exclusión, hostilidad y conflicto. Aunado a esto están los ciber ataques y la difamación, sin nunca conocer a tu victima verla cara a cara, pueden estar escondidos en un armario anónimo y el riesgo de ser castigado por calumnia se reduce al mínimo". (Bauman 2017 Pags 66-67)

Aquí la advertencia a la confianza líquida, el cuidado que debe tener la generación líquida al entregarse a la nube, a un sistema que contiene la posibilidad de cualquier reacción, positiva o negativa, reconocer los riesgos al entregar su cuerpo y vida privada a la nube.

"Esto esta causando una gran ansiedad ente los líquidos. La depresión y la ansiedad es mucho mayor en los modernidad liquida, pero la necesidad hedonista de la invisibilidad ha desaparecido. Sin embargo la invisibilidad podría ser la cura para estos males". (Baumna 2017 Pag 71) 
Las relaciones de los líquidos en el no espacio en un conflicto de intereses, la flexibilidad y lo inmediato provoca el deseo para no el amor, las relaciones en el no espacio buscan la libertad, pero sin sacrificar libertad no es posible tener seguridad "no puedes ganas libertad sin rendir algo de seguridad" (Baumna 2017 Pag 73)

Es un mundo nuevo, ni bueno ni malo solo debemos conocerlo para no caer en sus abismos, estar alertas abiertos y entender y confiar en la generación líquida.

\section{CONCLUSIONES}

Debemos comunicarnos con la generación líquida, aprender a movernos y mutar a su ritmo si es que queremos entendernos y como docentes estar a la altura de estos cambios. Docencia cambiante y activa, no crítica, analítica mas que nunca.

Cambio tecnológico y generacional "no sabemos a que llegara, si es positivo o negativo, reconocerlo y advertir de sus peligros," el sexting" en tender las consecuencias de hacer lo privado, publica y también reconocer su potencial y usarlo en nuestro beneficio.

Comprender y analizar las tendencias, el fenómeno de el "like", no banalizar los riesgos del cambio extremo de "look", analizar el mundo del tatuaje y de la cirugía plástica, banalidad, necesidad, moda o comercio .

La evolución y aceleración en la que los propios autores como Lipovetzy, Bauman, Byung Chul-Han o Rosa se han visto en su análisis de este fenómeno tecnológico y económico que nos ha afectado a todos

Abrazar las tecnologías, lo nano, los nuevos retos tecnológicos pero con un pensamiento crítico y desde el conocimiento de los posibles peligros de la economía rampante.

El derecho de actuar sobre el cuerpo femenino con conocimiento de la manipulación y las presiones del mass media para pertenecer a lo uniforme.

Retomar la ética y el equilibrio ante le comercio rampante y amoral.

Saber discernir ente la tecnología, la moral, la economía y evitar la anomia es el reto de los nacidos líquidos.

Los peores accidentes se dan con la velocidad y la generación líquida de mueve a la velocidad de la luz, equilibrar la velocidad con el pensamiento ético.

\section{FUENTES REFERENCIALES}

Bauman, Z. (2012). Amor Líquido. Acerca de la fragilidad de los vínculos humanos (2a . ed ) México: Fondo de Cultura Económica.

Bauman, Z. (2017). Born Liquid. Gran Bretaña: Polity.

Bauman, Z. (2009). Miedo Líquido (3a ed.). Barcelona: Paidos.

Criado M.A. (2019). 22 febrero 2019, La imprenta, la radio o al television, generaron sus propios famosos. El pais Sitio web: https://elpais.com/elpais/2019/02/20/ciencia/1550651024 330137.html

Han, B. C. (2016). La expulsión de lo distinto (2 2 ed.). Barcelona, Herder.

Hartmut, R. (2013). Social Acceleration. A new Theory of Modernity. Nueva York: Columbia University Press.

Laborde, A. (9 mayo de 2018). Una experiencia religiosa para lucir en Nueva York. El País, p. 16.

Lipovetsky, G. (2015). De la Ligereza. Barcelona: Anagrama.

Lipovetsky, G. (2005). Hypermodern Times. Cambridge, U.K.: Polity Press.

Waldrop, M. (2017). La teoría de la relatividad de Einstein explicada en cuatro simples pasos, noviembre 9, 2017, de National Geographic Sitio web: www.nationalgeographic.es/ciencia/2017/05/ 